\title{
L'aménagement hydraulique de la vallée de l'Orb en liaison avec l'usine de Montahut
}

\author{
Hydraulic development of the Orb Valley \\ and connection with the Montahut power station
}

\author{
PAR PH. DEYMIÉ
}

INGÉNIEUR EN CHEF DES PONTS ET CHAUSSÉES

\begin{abstract}
Le débit d'étiage, à Béziers, de l'Orb, dont le principal affluent est le Jaur, est de l'ordre de 500 litres-seconde, trés insuffisant pour permettre des irrigations pendant la saison sèche. L'usine de Montahut, qui utilisera la différence de niveau entre la haute vallée de l'Agont et la vallée du Jaur, amènera sur le versant méditerranéen un volume total de l'ordre de 173 millions de $m^{3}$ en année sèche, prélevé sur le versant atlantique.

Cet apport d'ean permettra d'emmagasiner, dans le réservoir du Rieuberlon situé sur un petit affuent rine droite de l'Orb, un volume d'eau d'environ 122 millions de $\mathrm{m}^{g}$ et d'irriguer ainsi 15.000 ha.
\end{abstract}

L'Orb prend naissance à la cote 825 , à la limite des départements de l'Aveyron et de l'Hérault, au nord-ouest de Lodève. En aval d'Avène, cette rivière se fraye un passage dans une gorge étroite, découpée dans les granits et les schistes métamorphiques. Après Bédarieux, l'Orb coule vers le sud-ouest jusqu'à son confluent avec le Jaur. Il change alors de direction, vers le sudest, et traverse les gorges de Vieussan dans les schistes et les grès siluriens avec, par endroits, des calcaires dévoniens. A Réals, l'Orb débouche à la cote 26, dans la zone de grande culture, et se jette dans la mer Méditerranée à Valras, après voir traversé Béziers. Sa longueur totale est de $136 \mathrm{~km}$ (fig. 1).

L'Orb est essentiellement une rivière à régime méditerrancen, avec un étiage très bas de l'ordre de $500 \mathrm{l} / \mathrm{s}$, et des crues violentes et rapides qui ont été évaluées à $3.500 \mathrm{~m}^{3}$ par seconde et dont la plus récente est celle du 6 décembre 1953.

La $5^{\circ}$ Circonscription Electrique à Toulouse a exploité :

- Une station de jaugeage sur l'Orb au Mas Blanc immédiatement en amont de Bédarieux;

\begin{abstract}
When the Orb, whose main tributary is the Jaur, is at its lowest level, the rate of flow at Beziers is about 500 litres per second which is insufficient for irrigation purposes during the dry season.

The Montahut power station which will male use of the drop in level between the upper Agout valley and the Jaur valley will bring about 173 million cabic metres of water in a dry year to the Mediterranean side of the watershed from the Atlantic side.

The vater made abailable in this way will make it possible to store about 122 million cubic metres in the Rienberlou reservoir situated on a right bank tributary in the Orb and thus enable 15,000 hectares to be irrigated.
\end{abstract}

- Une station de jaugeage sur le Jaur à Olargues.

Malheureusement, ces deux stations de jaugeage sont placées dans des sections où le lit de la rivière n'est pas fixé et les résultats des observations ne sont valables que pour une courte période, de 1920 à 1925 , et encore avec une grande marge d'incertitude.

Quoi qu'il en soit, ces jaugeages ont donné

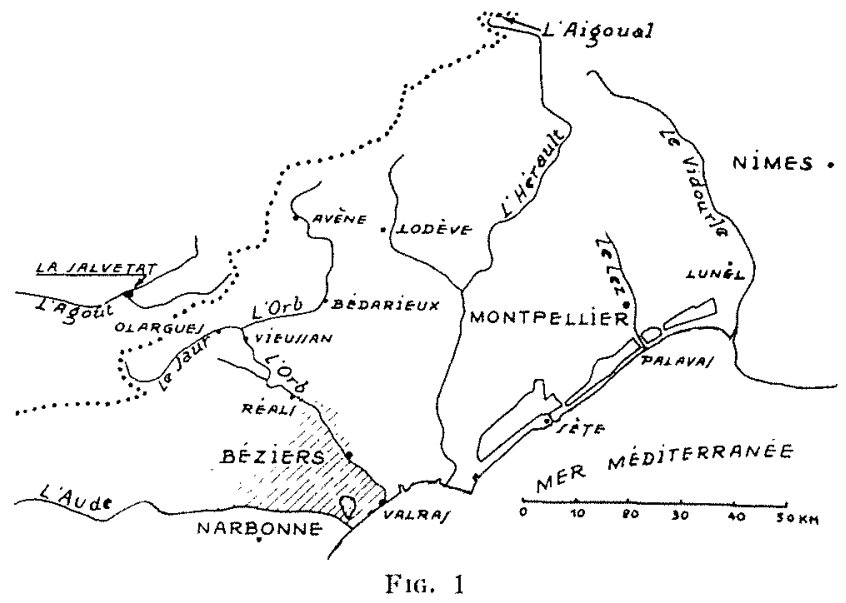


pour l'année 1924, année particulièrement sèche, en considérant l'année hydrologique du $1^{\text {er }}$ octobre au 30 septembre, les résultats suivants:

\begin{tabular}{|c|c|c|}
\hline \multirow{2}{*}{ Mors } & \multicolumn{2}{|c|}{ Débits moyens mensuels } \\
\hline & Orb $\left(\mathrm{m}^{3} / \mathrm{s}\right)$ & Jaur $\left(\mathrm{m}^{3} / \mathrm{s}\right)$ \\
\hline Octobre & 1,2 & 0,3 \\
\hline Novembre & 10,7 & 8,5 \\
\hline Décembre . & 11,8 & 5,1 \\
\hline Janvier . . & 3.1 & 1,1 \\
\hline Février & 4,1 & 0,9 \\
\hline Mars . . & 7,4 & 2,9 \\
\hline Avril & 18,4 & 14,8 \\
\hline Mai . & 11,7 & 8,5 \\
\hline Juin & 5,9 & 3,2 \\
\hline Juillet & 3,7 & 0,8 \\
\hline Août. & 1,8 & 0,2 \\
\hline Septembre.. & 1,0 & 0,3 \\
\hline
\end{tabular}

L'Orb et le Jaur sont incapables de fournir au fil de l'eau les débits nécessaires aux irrigations pendant les mois où ces' irrigations sont les plus nécessaires et n'assurent même pas en été les besoins actuels pour la salubrité publique et pour l'alimentation du Canal du Midi.

Pour cette même année 1924 et sous les réserves indiquées plus haut, les quantités d'eau qui auraient été susceptibles d'être emmagasinées pour l'irrigation auraient été les suivantes :

- pour le Jaur : 80 millions de mètres cubes en maintenant un débit réservé de $0,5 \mathrm{~m}^{3} / \mathrm{s}$; - pour l'Orb : 110 millions de mètres cubes en maintenant un débit réservé de $3 \mathrm{~m}^{3} / \mathrm{s}$.

Le Jaur est séparé de la vallée de l'Agoût par le massif de l'Espinouse qui marque en même temps la limite entre le versant méditerranéen et le versant atlantique.

Malgré le peu de distance qui les sépare, les pluviosités dans ces deux vallées sont très différentes.

Voici les moyennes des hauteurs de pluie enregistrées depuis 1873 , d'une part à La Salvetatsur-Agoût, d'autrè part à Béziers.

\section{La Salvetat-SUR-Agout}

Moyenne pendant les six mois d'hiver Moyenne pendant les six mois d'été..

$792 \mathrm{~mm}$ $536 \mathrm{~mm}$

Moyenne annuelle....... $1.328 \mathrm{~mm}$

\section{Station de Béziers}

Moyenne pendant les six mois d'hiver Moyenne pendant les six mois d'été.

$383 \mathrm{~mm}$ $248 \mathrm{~mm}$

Moyenne annuelle.......

$631 \mathrm{~mm}$
La pluviosité sur le versant atlantique est plus du double de la pluviosité sur le versant méditerranéen.

D'autre part, la vallée de l'Agoût en amont de La Salvetat est à la cote moyenne 750 , tandis que la vallée du Jaur dans' la région d'Olargues n'est qu'à la cote moyenne 140 (fig. 2).

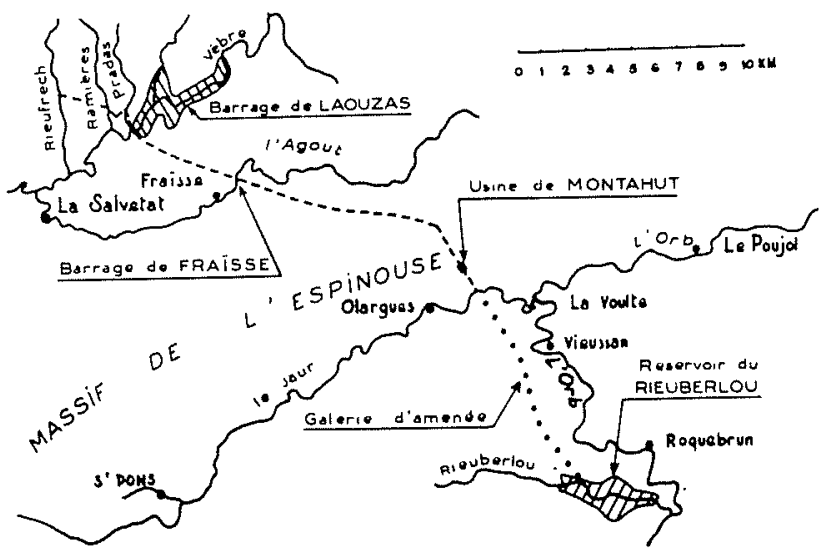

FIG. 2

Electricité de France a projeté d'utiliser cette différence de niveau en déversant les eaux emmagasinées dans la haute vallée de l'Agoût dans une usine située dans la vallée du Jaur, à Montahut, à l'aval d'Olargues.

Le barrage de Laouzas emmagasinera les eaux de la Vèbre et de trois affluents dans un réservoir de 40 millions de mètres cubes.

Une galerie d'amenée recevra au passage les eaux de l'Agoût à Fraisse et amènera l'ensemble des eaux dérivées à l'usine souterraine de Montahut.

Les caractéristiques de cette usine, d'après la demande de concession, sont les suivantes:

- Hauteur de chute : $625 \mathrm{~m}$.

- Débit équipé : $14 \mathrm{~m}^{3} / \mathrm{s}$.

-- Volume turbiné total : 173 millions de $\mathrm{m}^{3}$ en année sèche.

- Puissance : $67.000 \mathrm{~kW}$.

- Production annuelle moyenne : 238 millions de $\mathrm{kW}$.

Cette usine amènera donc dans la vallée du Jaur et de l'Orb, en année sèche, un volume d'environ 173 millions de mètres cubes, du même ordre de grandeur que celui pouvant être fourni par ces deux rivières réunies.

Cette usine fonctionnera comme usine de pointe surtout pendant les mois de novembre à mai, c'est-à-dire pendant les mois où, sauf avril et mai, il n'y aura pas d'irrigation. 


\section{*:*}

Le problème est donc d'emmagasiner le plus grand volume possible pendant la période de fonctionnement de l'usine, pour le restituer pen. dant la période d'irrigation.

Il existe dans les gorges de l'Orb, autour de Vieussan, plusieurs sites de barrages qui ont l'inconvénient, tout en ayant une capacité assez limitée, de submerger les lieux habités et les seules terres cultivables de cette région, situées dans le fond de la vallée. En outre, l'imperméabilité de ces réservoirs n'est pas assurée.

Plus en aval, la cuvette du Rieuberlou, située sur un petit affluent rive droite de l'Orb dans les schistes et grès imperméables, a été étudiée par M. Silhol, ingénieur des Travaux Publics de l'Etat à Béziers. Les terres constituant cette cuvette sont peu cultivées (140 ha sur 375 ha). Deux maisons seulement seront noyées. L'apport solide sera à peı̣ près nul et le débit de crue du Rieuberlou $\left(350 \mathrm{~m}^{3} / \mathrm{s}\right)$ sera relativement aisé à évacuer.

Le Service des Ponts et Chaussées, ou plus exactement le Service hydraulique, a établi en 1953-1954 un projet utilisant ce réservoir pour l'irrigation en s'attachant à rendre le fonctionnement de ce réservoir indépendant du fonctionnement de l'usine de Montahut.

A la cote 140 , ce réservoir aura une capacité de 85 millions de mètres cubes, la crête du barrage principal étant à la cote 142 , ce qui donnera à ce barrage une hauteur hors sol d'environ $70 \mathrm{~m}$; la possibilité de construire un barragevoûte n'est pas exclue.

$$
\text { **: }
$$

Des barrages secondaires barreraient les cols situés autour de la cuvette :

Col des Eucalyptus.. hauteur hors sol : $28 \mathrm{~m}$ Col du Cabal....... hauteur hors sol : $18 \mathrm{~m}$ Col des Arbousiers. hauteur hors sol : $8 \mathrm{~m}$

Ce réservoir serait alimenté par une galerie d'amenée ayant une section en fer à cheval de $2,70 \mathrm{~m}$ de diamètre et de $6 \mathrm{~m}^{2}$ de surface (section économique permettant le travail mécanique), une pente de $0,86 \mathrm{~m}$ par $\mathrm{km}$, une longueux de $9.300 \mathrm{~m}$, et capable d'un débit de $10,9 \mathrm{~m}^{3} / \mathrm{s}$.

Cette galerie aurait son origine sur le Jaur, à l'aval du canal de fuite de l'usine de Montahut, la prise d'eau étant assurée par un barrage à la cote 148 déterminée, avec une marge de sécurité, d'après la cote de restitution de l'usine (150).

Le débit dérivé par cette galerie serait donc égal à 10,9/14 du débit maximum turbiné.

Sur ces bases, et d'après les renseignements fournis' par Electricité de France sur les volumes d'eau turbinés mensuellement en année relativement sèche (type 1934), l'exploitation du réservoir du Rieuberlou serait schématisée dans le tableau suivant:

\begin{tabular}{|c|c|c|c|c|}
\hline Mois & 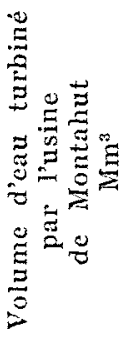 & 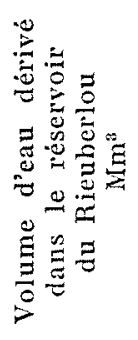 & 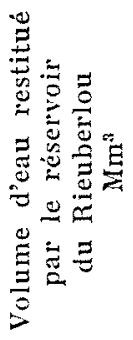 & 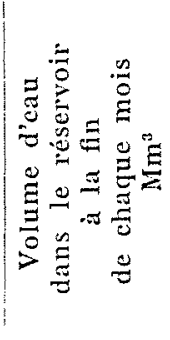 \\
\hline Octobre & 28 & 2.1 & & 2,1 \\
\hline Novembre & 14,8 & 11,2 & - & 13,3 \\
\hline Décembre & 30.4 & 23,6 & - & 36,9 \\
\hline Janvier . . . & 37,5 & 29,1 & - & 66,0 \\
\hline Février ... & 22,6 & 17,5 & $\ldots$ & 83,5 \\
\hline Mars ... & 14,9 & 1,5 & — & 85,0 \\
\hline Avril ... & 27,2 & 20,0 & 20,0 & 85,0 \\
\hline Mai ... & 20,5 & 15,9 & 20,6 & 80,3 \\
\hline Juin & 1,2 & 0,9 & 20,0 & 61,2 \\
\hline Juillet & 1,1 & 0,8 & 20,6 & 41,4 \\
\hline Août . & 0,1 & 0 & 20,6 & 20,8 \\
\hline \multirow[t]{2}{*}{ Septembre. } & 0 & 0 & 20,0 & 0,8 \\
\hline & 173,1 & 122,6 & 121,8 & \\
\hline
\end{tabular}

Le volume d'eau ainsi restitué pour l'irrigation s'élèverait à 122 millions de mètres cubes par an, supérieur à la capacité du réservoir ( 85 millions de $\mathrm{m}^{3}$ ) parce que pendant les mois d'avril et de mai, il y aurait à la fois apport dans le réservoir de 36 millions de mètres cubes et restitution par le réservoir de 40 millions de mètres cubes.

Il est possible que le débit maximum turbiné indiqué ci-dessus dans une première étude d'Electricité de France $\left(14 \mathrm{~m}^{3} / \mathrm{s}\right)$ soit augmenté.

Dans cette hypothèse, pour éviter que cette augmentation du débit turbiné entraîne une augmentation de la section de la galerie, il conviendra de prévoir sur le Jaur un barrage plus important qui constituera une réserve supplémentaire et dont la tranche supérieure assurera, en même temps, une compensation journalière, le volume restitué par l'usine de Montahut ne devant pas dépasser en général un million de mètres cubes par jour.

Ce barrage serait probablement situé à La Voulte et la longueur de la galerie d'amenće serait diminuée de $1,5 \mathrm{~km}$.

Le fonctionnement du réservoir du Rieuberlou serait ainsi indépendant du fonctionnement de l'usine de Montahut.

En comptant $8.000 \mathrm{~m}^{3}$ d'eau par hectare, ces 122 millions de mètres cubes permettront d'irriguer au moins 15.000 ha, c'est-à-dire les terres cultivées' de la vallée de l'Orb à partir du Rieuberlou et les terres qui s'étendent vers Capestang et vers l'Aude. 


\section{DISCUSSION}

Président : M. BengenoN

Répondant à M. Quesnes, qui trouve trop élevées les estimations données pour les quantités d'eau susceptibles d'être emmagasinées avec les seuls apports du Jaur et de l'Orb, M. Deysiné répond que les calculs ont été effectués par M. Silhol, Ingénieur des T.P.E. à Béziers, d'après les débits fournis par la $5^{\mathrm{e}}$ Circonscription Electrique de Toulouse et présentent donc les mêmes incertitudes que ces derniers.

Répondant à une question de M. Tissier, M. Deymié précise que, dans l'hypothèse où il s'est placé, le barrage de prise d'eau, en l'espèce le barrage de la Voulte, accueillerait évidemment toutes les eaux, y compris celles du Jaur, avec celles qui proviendraient de l'usine de Montahut. Il a essayé de voir si les eaux du Jaur suffiraient au fonctionnement du réservoir de Ricuberlou, mais elles n'y suffisent pas.

M. le Président remercie M. l'Ingénieur en Chef Dexmé de ses deux intéressantes communications.

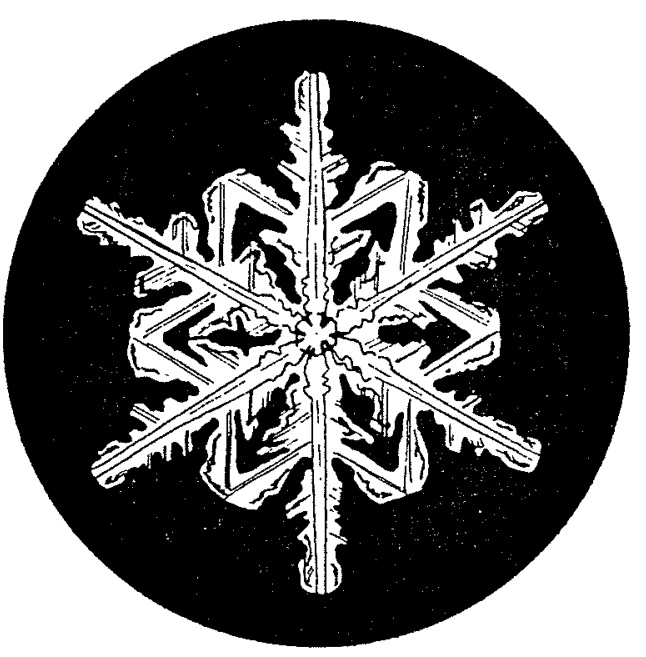

